\title{
A Significance of Sodium-Glucose Cotransporter 1 (SGLT1) Inhibition by SGLT2 Inhibitors
}

\section{Hidekatsu Yanai}

Department of Internal Medicine, National Center for Global Health and Medicine Kohnodai Hospital, Chiba, Japan

Sodium-glucose cotransporter 2 (SGLT2) is expressed in the proximal tubule of kidney and mediates reabsorption of approximately 90 percent of glucose [1]. A newly developed anti-diabetic drug, the SGLT2 inhibitor blocks reabsorption of glucose by inhibiting SGLT2, increasing renal excretion of glucose without requiring insulin action [2]. Various clinical trials showed that SGLT2 inhibitors improved the glycemic control as monotherapy and combination therapy with anti-diabetic drugs [3,4]. Since April 2014, ipragliflozin, dapagliflozin, luseogliflozin, canagliflozin, tofogliflozin and empagliflozin have been approved and widely used to date in Japan.

Recently, I reported the review article entitled "Sodium-Glucose Cotransporter 2 Inhibitors: Possible Anti-Atherosclerotic Effects beyond Glucose Lowering" [5]. In the review, SGLT-2 inhibitors seem to be associated with improvement of glucose control, body weight and blood pressure by a relatively large number of studies. Such favorable effects of SGLT2 inhibitors on metabolic parameters seem to be due to the class effect of SGLT2 inhibitors, because SGLT-2 inhibitors have similar chemical structures.

If it is true, is there a significance of SGLT1 inhibition by SGLT2 inhibitors? Currently, several SGLT2 inhibitors with various degrees of selectivity toward SGLT2 versus SGLT1 selectivity are being tested in clinical trials [4]. One of SGLT2 inhibitors, canagliflozin, has been reported to have a low potency to inhibit SGLT1 [6], which is highly distributed in intestine. Therefore, canagliflozin may improve postprandial hyperglycemia due to blocking intestinal absorption of glucose by inhibiting SGLT1. Recently, a potent and highly selective SGLT2 inhibitor, tofogliflozin, was identified [7].

I added daily $20 \mathrm{mg}$ of tofogliflozin to vildagliptin (100mg/day), pioglitazone $(30 \mathrm{mg} /$ day), metformin $(2000 \mathrm{mg} /$ day) and stopped glimepiride $(0.5 \mathrm{mg} /$ day $)$ as the treatment for a type 2 diabetic patients. In spite of discontinuation of glimepiride, $\mathrm{HbAlc}$ decreased from $8.0 \%$ to $7.6 \%$ (after one month), 7.4\% (after 2 months). However, HbAlc increased to $7.9 \%$ at 4 months after the start of tofogliflozin. I switched from tofogliflozin to canagliflozin $(100 \mathrm{mg} /$ day). HbAlc increased to $9.1 \%$ at 2 months after the switching to canagliflozin, and I again switched from canagliflozin to tofogliflozin. HbAlc decreased to $8.9 \%$ at 5 weeks after the switching to tofogliflozin.

A significance of SGLT1 inhibition and selectivity for SGLT2 are the issues that cannot be ignored. We should study how SGLT1 inhibition and selectivity for SGLT2 influence on glucose control, and we should also elucidate whether effect of these issues is different depending on the patients or not.

\section{References}

1. Vallon V, Platt KA, Cunard R, Schroth J, Whaley J, et al. (2011) SGLT2 mediates glucose reabsorption in the early proximal tubule. J Am Soc Nephrol 22: 104-112.

2. Jabbour SA Goldstein BJ (2008) Sodium glucose co-transporter 2 inhibitors: blocking renal tubular reabsorption of glucose to improve glycaemic control in patients with diabetes. Int J Clin Pract 62: 1279-1284.

\section{Publication History:}

Received: December 25, 2015

Accepted: December 28, 2015

Published: December 31, 2015

\section{Keywords:}

Sodium-Glucose cotransporter 2, Glucose control, Anti-diabetic drugs

3. Clar C, Gill JA, Court R, Waugh N (2012) Systematic review of SGLT2 receptor inhibitors in dual or triple therapy in type 2 diabetes. BMJ Open 2.

4. Musso G, Gambino R, Cassader M, Pagano G (2012) A novel approach to control hyperglycemia in type 2 diabetes: sodium glucose co-transport (SGLT) inhibitors: systematic review and meta-analysis of randomized trials. Ann Med 44: 375-393.

5. Yanai H, Katsuyama H, Hamasaki H, Adachi H, Moriyama S, et al. (2016) Sodium-Glucose Cotransporter 2 Inhibitors: Possible Anti-Atherosclerotic Effects Beyond Glucose Lowering. J Clin Med Res 8: 10-14.

6. Scheen AJ, Paquot N (2014) Metabolic effects of SGLT-2 inhibitors beyond increased glucosuria: A review of the clinical evidence. Diabetes Metab 40: S4-4S11.

7. Ohtake Y, Sato T, Kobayashi T, Nishimoto M, Taka N, et al. (2012) Discovery of tofogliflozin, a novel C-arylglucoside with an O-spiroketal ring system, as a highly selective sodium glucose cotransporter 2 (SGLT2) inhibitor for the treatment of type 2 diabetes. J Med Chem 55: 7828-7840.
"Corresponding Author: Dr. Hidekatsu Yanai, Department of Internal Medicine, National Center for Global Health and Medicine Kohnodai Hospital, 1-7-1 Kohnodai, Chiba, 272-8516, Japan; E-mail: dyanai@hospk.ncgm.go.jp

Citation: Yanai H (2015) A Significance of Sodium-Glucose Cotransporter 1 (SGLT1) Inhibition by SGLT2 Inhibitors. Int J Diabetes Clin Diagn 1: 102. doi: http://dx.doi.org/10.15344/2394-1499/2015/111

Copyright: (c) 2015 Yanai et al. This is an open-access article distributed under the terms of the Creative Commons Attribution License, which permits unrestricted use, distribution, and reproduction in any medium, provided the original author and source are credited. 